\title{
Intra-hospital mortality of stroke and its predictive factors in a reference hospital in Ouagadougou, Burkina Faso
}

\author{
Lompo Djingri Labodi ${ }^{1}$, Cisse Kadri ${ }^{3}$, Yameogo Nobila Valentin ${ }^{2}$, Napon Christian ${ }^{2}$ and Kabore B Jean ${ }^{2}$ \\ ${ }^{1}$ University Hospital of Tingandogo, Unit of Formation and Research of the Sciences of the Health, University Ouaga I-Pr Joseph Ki-Zerbo \\ ${ }^{2}$ University Hospital of Yalgado Ouedraogo of Ouagadougou, Unit of Formation and Research of the Sciences of the Health, University Ouaga I-Pr Joseph Ki-Zerbo \\ ${ }^{3}$ Institute of Research in Health Sciences, Ouagadougou, Department of Medical Biology and Public Health
}

\begin{abstract}
Introduction: The aim of this study was to identify predictive factors for intra-hospital stroke mortality in a cohort of patients hospitalized for stroke within 72 hours of the onset of signs at the Tingandogo University Hospital in Ouagadougou, (Burkina Faso).

Patients and methods: It was a prospective, analytical study of consecutive patients hospitalized for stroke from March 2015 to February 2016 . Sociodemographic characteristics, vascular risk factors, comorbidities, clinical, neuroradiological and biological data at admission, as well as Intra-hospital mortality rates have been described. An univariate, then multivariate analysis with logistic regression allowed to identify the independent predictors of intra-hospital mortality of stroke.

Results: During the study period, 157 patients were consecutively hospitalized for stroke, 113 cases of cerebral infarction (72\%) and 44 cases of intracerebral hemorrhage (28\%). The male patients accounted for $61.1 \%$ of the workforce, the average age was 61.8 years (range 28-92 years). At admission, the National Institute of Health Stroke Score (NIHSS) averaged 15 (range 0-38), neurological deficit was severe (NIHSS $\geq 17$ ) in 46.7\%, Glasgow admission score averaged 13.7 (extremes 3 and 15$)$ and 12 patients (7.6\%) were in a coma (Glasgow $\leq 8)$. The mean hospital stay was 13.2 days (range 3 and 40 days). The intra-hospital mortality rate was $28.7 \%$ with an average intra-hospital death rate of 12.1 days (range 3 and 40 days). Independent predictive factors for intra-hospital mortality were NIHSS at admission $\geq 17$ (OR 2.909, $\mathrm{p}=0.036)$, admission hyperglycemia (OR 6.752, $\mathrm{p}=0.000)$, renal failure at admission $(\mathrm{OR} 3.903, \mathrm{p}=0.031)$, hemorrhagic stroke $(\mathrm{OR}$ 5.580, $\mathrm{p}=$ 0.003 ) and cardiac comorbidities (OR 6.393, p = 0.009). Intra-hospital mortality is high in Tingandogo University Hospital in Ouagadougou, Burkina Faso. Reasons for the increased mortality rate have been discussed.
\end{abstract}

Conclusion: Intra-hospital mortality of stroke remains high in Tingandogo University Hospital. Reducing early mortality by stroke in sub-Saharan hospitals requires early access to the highest number of patients to quality care in high quality specialized facilities such as stroke units.

\section{Introduction}

Stroke appears today as a major cause of preventable death and disability in the world [1,2]. Today, early intra-hospital or monthly mortality per stroke varies from less than $15 \%$ to $22.9 \%$ in Western countries [3-5]. Early mortality of cerebral infarcts has declined sharply, dropping from $10.2-12.7 \%$ in 2003 to $8.4-10.1 \%$ in 2013 , as a result of improved access to quality care, including timely patient transportation, evidence-based medical interventions, and specialized high-quality facilities such as stroke units (SU) [6]. About Intracerebral haemorrhage (ICH), their intra-hospital mortality has remained quasistationary [7] due to the absence of specific therapeutics effective in reducing mortality [8].

Developing countries account for nearly $85 \%$ of stroke deaths worldwide $[1,2]$ and intra-hospital mortality rates or one month per stroke range from 25 to $46 \%$ [9]: 24. 10\% in Senegal [10]; 25\% in Congo-Brazzaville [11]; 26. 8\% respectively in Cameroon and Uganda [12-14]; 35 to 46 per cent in Nigeria [15,16], except for a South African study that reported a 3 -month mortality rate of $22.5 \%$ [17].

This high stroke mortality rate in sub-Saharan Africa may be due to the higher proportion of ICHs in blacks [18] and their high lethality around the world, the high incidence of severe or catastrophic stroke $[19,20]$, the shortage and prohibitive costs of medico-technical equipment, inadequate health human resources $[21,22]$. Many predictive characteristics of early stroke death have been identified $[14,23,24]$. Hospital mortality of stroke is an excellent tool for measuring performance and quality of hospital management of stroke; its evaluation and a better understanding of its predictive factors are useful for the implementation of specific therapies and effective strategies for the management of high-risk patients $[4,25]$, in a resource-constrained environment. To our knowledge, no studies have yet been made in Burkina Faso on the intra-hospital mortality of stroke and its predictive factors of occurrence.

The purpose of this study was to evaluate intra-hospital mortality and to identify independent predictive factors for intra-hospital deaths of patients hospitalized with stroke less than 72 hours.

\section{Patients and methods}

This is a prospective, transverse, observational, descriptive and analytical study carried out at the University Teaching Hospital (UTH)

Correspondence to: Lompo Djingri Labodi, University Hospital of Tingandogo, Unit of Formation and Research of the Sciences of the Health, University Ouaga I-Pr Joseph Ki-Zerbo, Burkina Faso; Tel : +226 702398 34; E-mail: labodilompo@yahoo.fr

Key words: stroke, mortality, predictive factors

Received: August 20, 2017; Accepted: September 25, 2017; Published: September 27, 2017 
of Tingandogo in Ouagadougou. It is one of the tertiary hospitals (3rd reference level) of the city of Ouagadougou, political capital of Burkina Faso. The hospital has 600 beds in 15 pavilions, of which only 200 are operational at the present stage. Our study was conducted in the department of neurology, housed in the department of medicine and medical specialties, which has 34 beds distributed in 12 rooms. Our study lasted 18 months, from March 1, 2015 to August 30, 2016. It examined adult patients ( $>16$ years of age) of all sexes, consecutively hospitalized in the Department of Neurology, for neuroimaging (CT or Brain MRI) confirmed stroke, up to 72 hours after its onset, excluding subarachnoid haemorrhages. For each patient, the following tests were performed at admission: blood pressure (BP), temperature, capillary blood glucose; National Institutes of Health Stroke Score (NIHSS), Glasgow coma score (GCS); Electrocardiogram (ECG) standard; brain scan; creatininemia \& blood urea, numeration blood formula-platelet levels, blood crest, blood ionogram. In case of cerebral infarction, the lipid balance (total cholesterol, Low Density Lipoprotein (LDL) cholesterol, High Density Lipoprotein (HDL) cholesterol, triglycerides), transthoracic heart ultrasound and holter ECG were also performed, if necessary. The radiologists performed the interpretation of the brain scan. Additional tests were performed on a case-by-case basis: standard chest $\mathrm{x}$-ray in case of suspicion of bronchopneumopathy, biological check-ups if necessary; tick drop in case of suspicion of malaria, cytobacteriological examination of the urine if suspicion of urinary infection, blood culture, in case of suspicion of septicemia; brain scanner if needed, ...

The evolution of the patients during hospitalization was monitored daily by a clinical evaluation possibly aided by complementary examinations on a case by case basis and the complications regularly noted in the medical file, until the end of the hospitalization. The complications sought included those observed at admission or which appeared during hospitalization. Upon discharge from hospital, patients were subdivided according to discharge status, surviving patients and deceased patients.

Patients were treated according to the recommendations of the European Stroke Organization (ESO 2008). The Department of Neurology does not yet have SU.

The variables studied included socio-demographic characteristics, vascular risk factors (VRFs), modified Rankin score (mRS) before stroke, comorbidities, care pathway, (temperature, BP, GCS, NIHSS), radiographic data on initial CT scan [nature of stroke, ICH volume, other neuroradiological abnormalities), qualitative biological data at admission (hemoglobin, leukocytes, blood sugar, serum sodium, serum potassium, serum creatinine); medical complications, present at admission or occurring during hospitalization (thromboembolic venous complications, haemorrhagic complications, metabolic complications, infectious complications, cardiac complications, respiratory complications, neurological complications present on admission or appearing during hospitalization: neurological deterioration during hospitalization (increased neurological deficit, including alertness), epileptic seizures, relapse or extension of stroke, life-threatening prognosis at the end of hospitalization ( survivors / deceased).

The consent of the patients or that of their legal representatives was ensured before the recruitment. The study protocol was approved by the ethics committee of the University of Ouaga I-Pr Joseph KIZERBO and by the national ethics committee of Burkina Faso.

Statistical analyzes were carried out using the SPSS12 software. Student's t-test was used to compare the averages and the Pearson Chi- square test to compare percentages; the value of $\mathrm{p}<0.05$ was considered as a threshold of statistical significance. Univariate analyzes between the different characteristics of the patients and intra-hospital mortality made it possible to select the variables significantly associated with intra-hospital mortality. Finally, multiple logistic regression analysis identified independent factors influencing intra-hospital mortality. Only variables with a $\mathrm{p}<0.20$ value in bivariate analysis were taken into account for multivariate analysis.

\section{Results}

During the study period, we recorded 157 cases of stroke, 113 cases of cerebral infarction $(72 \%)$ and 44 cases of ICH $(28 \%)$. There were 61 female patients $(38.9 \%)$, a sex ratio of 1.57 . The mean age of the patients was 61.8 years (range 28 and 92 years); the majority of patients $(54.8 \%)$ were $\leq 65$ years of age; $67.5 \%$ of patients had no education; $68.2 \%$ of the patients resided in urban areas; 112 patients $(73.3 \%)$ were referred from a health facility, while 45 patients $(28.7 \%)$ consulted directly; the majority of patients $(83.4 \%)$ consulted within $\leq 24 \mathrm{~h}$; for $75.2 \%$ of patients, CT was performed within 12 hours after arrival in the emergency room. At least one VRF was found in 131 patients, either $83.4 \%$.

At admission, 90 patients $(57.3 \%)$ had NIHSS $\leq 16$ (mild to moderate neurological deficit) versus 67 (42.7\%) patients with NIHSS> 16 (severe to very severe neurological deficit), 110 patients $(70.1 \%)$ had a normal vigilance state, 35 patients $(22.3 \%)$ had altered vigilance and 12 patients (7.6\%) were in a coma. Anomalies of clinico-biological constants at admission were dominated by hypertension with 111 cases (70.7\%), hyperglycemia with 71 cases $(45.2 \%)$, and hyperleukocytosis with 45 cases $(28.7 \%)$. The initial volume of HIC was $\leq 60 \mathrm{cc}$ in 31 patients $(70.5 \%)$ and $>60 \mathrm{cc}$ in 13 patients (29.5\%). General and neurological complications present at admission or appearing during hospitalization were dominated by fever with 85 cases $(54.5 \%)$, pneumonia with 75 cases $(48.7 \%)$, neurological deterioration with 60 cases (38.9\%) and epileptic seizures with 27 cases (17.8\%).

Table 1 shows the distribution of patients according to their main characteristics.

The mean hospital stay was 13.2 days (range 3 and 40 days). At the end of the hospitalization, 45 patients died, ie an intra-hospital stroke mortality rate of $28.7 \%$ for an average life expectancy after stroke in the 12.1 days (extremes 3 and 40 days) and an average hospital stay of 13.7 days (extremes 4 and 33 days) in survivors $(p=0.019)$. Depending on the nature of the stroke, there were 26 cases of cerebral infarction deaths and 19 cases of HIC deaths, ie intra-hospital fatality rates, $23 \%$ for infarcts and $43.2 \%$ for the ICHs $(p=0.012)$. The intra-hospital mortality rate was $12.7 \%$ at day $7,21 \%$ on day 14 and $28.7 \%$ at the end of hospitalization.

At the end of hospitalization, there were 112 survivors (71.3\%), of which 39 patients $(34.8 \%)$ were independent or autonomous (mRS $\leq 2)$.

After univariate analysis, cardiac comorbidities, an admission delay of $>24$ hours, a time to perform CT $\leq 4$ hours, initial clinical severity of stroke (NIHSS $\geq 17$ ), intake swallowing disorders, coma on admission (GCS $\leq 8)$, intake fever, intake hyperglycemia, intake dyskalaemia, intake dysnatraemia , renal failure at admission, intake leukocytosis, hemorrhagic nature of stroke, were the variables significantly associated with intra-hospital mortality.

After multivariate analysis using the ascending logistic regression method, the following independent intra-hospital mortality predictive 


\begin{tabular}{|c|c|c|}
\hline Subject & Numbers & Frequencies \\
\hline \multicolumn{3}{|l|}{ Vascular risk Factors } \\
\hline High Blood Pressure (HBP) & 119 & $75.8 \%$ \\
\hline Hypercholestérolemia & 41 & $26.1 \%$ \\
\hline History of stroke & 34 & $21.7 \%$ \\
\hline Diabetes mellitus & 20 & $12.7 \%$ \\
\hline Alcoholism & 12 & $7.6 \%$ \\
\hline Smoking & 10 & $6.4 \%$ \\
\hline Obesity & 8 & $5.1 \%$ \\
\hline Comorbidities & 47 & $29.9 \%$ \\
\hline Pre-existing handicap & 8 & $5.1 \%$ \\
\hline \multicolumn{3}{|l|}{ Clinical data at admission } \\
\hline \multicolumn{3}{|l|}{$\begin{array}{l}\text { NIHSS } \\
\text { - Mean : } 15,01 \\
\text { - Extremes : 0-38 }\end{array}$} \\
\hline \multicolumn{3}{|l|}{$\begin{array}{l}\text { GCS } \\
\text { - Mean : 13,70 } \\
\text { - Extremes : 3-15 }\end{array}$} \\
\hline Coma $(\mathrm{GCS} \leq 8)$ & 12 & $7.6 \%$ \\
\hline HBP & 111 & $70.7 \%$ \\
\hline Hyperthermia & 23 & $14.6 \%$ \\
\hline \multicolumn{3}{|l|}{ biological intake data } \\
\hline Hyperglycemia & 71 & $45.2 \%$ \\
\hline Hyperleukocytosis & 45 & $28.7 \%$ \\
\hline Hypokaliemia & 39 & $25.3 \%$ \\
\hline Renal failure & 31 & $19.7 \%$ \\
\hline Hyponatremia & 23 & $15 \%$ \\
\hline Hypernatremia & 15 & $9.8 \%$ \\
\hline Anemia & 14 & $9.1 \%$ \\
\hline \multicolumn{3}{|l|}{ CT scan data at admission } \\
\hline Leucoaraiosis & 66 & $42 \%$ \\
\hline Cicatricial lesions & 37 & $23.6 \%$ \\
\hline $\begin{array}{l}\text { Early signs of cerebral } \\
\text { ischemia }\end{array}$ & 10 & $8.9 \%$ \\
\hline \multicolumn{3}{|l|}{$\begin{array}{l}\text { Initial volume of ICH } \\
\text { - Mean : } 43.9 \mathrm{cc} \\
\text { - Extreme } 18-91 \mathrm{cc}\end{array}$} \\
\hline Cerebral edema & 44 & $28 \%$ \\
\hline Brain swelling & 33 & $21 \%$ \\
\hline Hemorrhagic transformation & 20 & $17.7 \%$ \\
\hline Malignant sylvian infarction & 15 & $13.2 \%$ \\
\hline Ventricular flood of ICH & 24 & $54.5 \%$ \\
\hline \multicolumn{3}{|c|}{ Medical and neurogical complictions at admission and during hospitalisation } \\
\hline Fever & 84 & $54.5 \%$ \\
\hline Pneumopathy & 75 & $48.7 \%$ \\
\hline Neurological deterioration & 61 & $38.9 \%$ \\
\hline Epileptic seizures & 28 & $17.8 \%$ \\
\hline Cardiac complications & 25 & $16.2 \%$ \\
\hline Malaria & 21 & $13.6 \%$ \\
\hline Urinary tract infections & 20 & $13 \%$ \\
\hline $\begin{array}{l}\text { Venous thrombo embolic } \\
\text { complications }\end{array}$ & 10 & $6.4 \%$ \\
\hline Respiratory complications & 7 & $4.5 \%$ \\
\hline Sepsis & 6 & $3.9 \%$ \\
\hline Hemorrhagic complications & 5 & $3.2 \%$ \\
\hline Recurrence of stroke & 3 & $1.9 \%$ \\
\hline
\end{tabular}

Table 1. Distribution of patients according to the main characteristics at admission and / or during hospitalization

factors were identified: cardiac comorbidities (OR 6.393, 95\% CI 1.591-25.694, $\mathrm{p}=0.009$ ), cardiac comorbidity NIHSS $\geq 17$ (severe neurological deficit) on admission (OR 2.909, 95\% CI 1.071-7.900, $\mathrm{p}$ = 0.036), haemorrhagic stroke (OR 5.580, 95\% CI 1.822-17.091, $\mathrm{p}=$ 0.003 ), admission hyperglycemia (OR 6.752, 95\% CI 2.432-18.744, p
$=0.000$ ), renal insufficiency at admission (OR 3.903, 95\% CI 1.131 13.475); $\mathrm{p}=0.031$ ). The results of the univariate and multivariate analysis are summarized in the following Table 2.

\section{Discussion}

\section{Intra-hospital mortality}

The intra-hospital mortality rate of $28.7 \%$ found in this study remains high, but it is comparable to the results of the recent African sub-Saharan stroke studies, where intra-hospital mortality rates ranged from $25 \%$ to $46 \%[9,11,16]$ : $24.10 \%$ in Senegal [10]; $25 \%$ in CongoBrazzaville [11]; 26.8\% in Cameroon and Uganda respectively [12-14], $41 \%$ in the Gambia [26]; 35 to $46 \%$ in Nigeria [15,16,22]. However, in a South African study, the mortality rate at 3 months post-stroke was $22.5 \%[17]$.

Intra-hospital mortality in our study is, however, significantly higher than that reported in developed countries, where it varies from less than $15 \%$ to $22.9 \%[3-5,17,18,27]$. The lowest rates are for cerebral infarcts, whose mortality dropped from $10.2-12.7 \%$ to $8.4-10.1 \%$ between 2003 and 2013. The early mortality rate for ICHs has remained virtually unchanged [16]: $34 \%$ in France [27], $40 \%$ in the USA [8], because no specific therapies have yet proved effective in reducing mortality after ICH [8]. Reduction of early mortality in ischemic stroke patients in developed countries is due to improved access to quality care, including timely patient transportation, evidence-based medical interventions and specialized facilities of high quality such as SU [6]. Moreover, better prevention of vascular risk factors is more effective, which has reduced the incidence of catastrophic stroke still too frequent in Africa $[1,19,20]$. Conversely, the high mortality rate of stroke in sub-Saharan Africa may be due to a number of factors: the highest proportion of ICHs in blacks [18], the highest ICH lethality reported worldwide $[18,27]$; inadequate primary prevention of stroke $[19,20]$, the shortage and prohibitive costs of medical-technical equipment, and the inadequacy of qualified human resources for investigations, emergency care and rehabilitation of stroke patients in sub-Saharan Africa [28].

\section{Independent predictors of intra-hospital mortality}

Our study identified several independent predictors of intrahospital mortality after stroke.

The initial clinical severity of stroke (initial NIHSS $\geq 17$ ) recognized as an independent predictor of early mortality by our study and several other studies [13-15], directly reflect the extent and severity of neurological damage secondary to stroke $[3,13,14,27]$. We have shown, like other studies [13,14,29-32], that admission hyperglycemia was a predictor of early post-stroke mortality. Indeed, hyperglycemia often complicates severe acute strokes, in response to the major stress triggered by lesions of extensive cerebral necrosis, which explains the pejorative life prognosis in these patients. Hyperglycaemia, in turn, contributes to an aggravation of the initial infarction, via its toxicity in the ischemic penumbra zone and potentiation of early reperfusion lesions; the ultimate consequence is an increase in the final volume of the cerebral infarction, a greater risk of hemorrhagic transformation and a pejorative functional and vital prognosis [29-32]. Some studies have reported that hyperglycemia is associated with a poor prognosis after ICH but the underlying mechanisms have not yet been identified $[33,34]$. Onset, vigilance, severity of neurological deficit and blood glucose levels identify subgroups of patients at increased risk of early stroke death to ensure appropriate monitoring and care ideally in an intensive care stroke unit. 
Labodi LD (2017) Intra-hospital mortality of stroke and its predictive factors in a reference hospital in Ouagadougou, Burkina Faso

Table 2. Results of the uniivariate and multivariate analysis using the ascending logistic regression method.

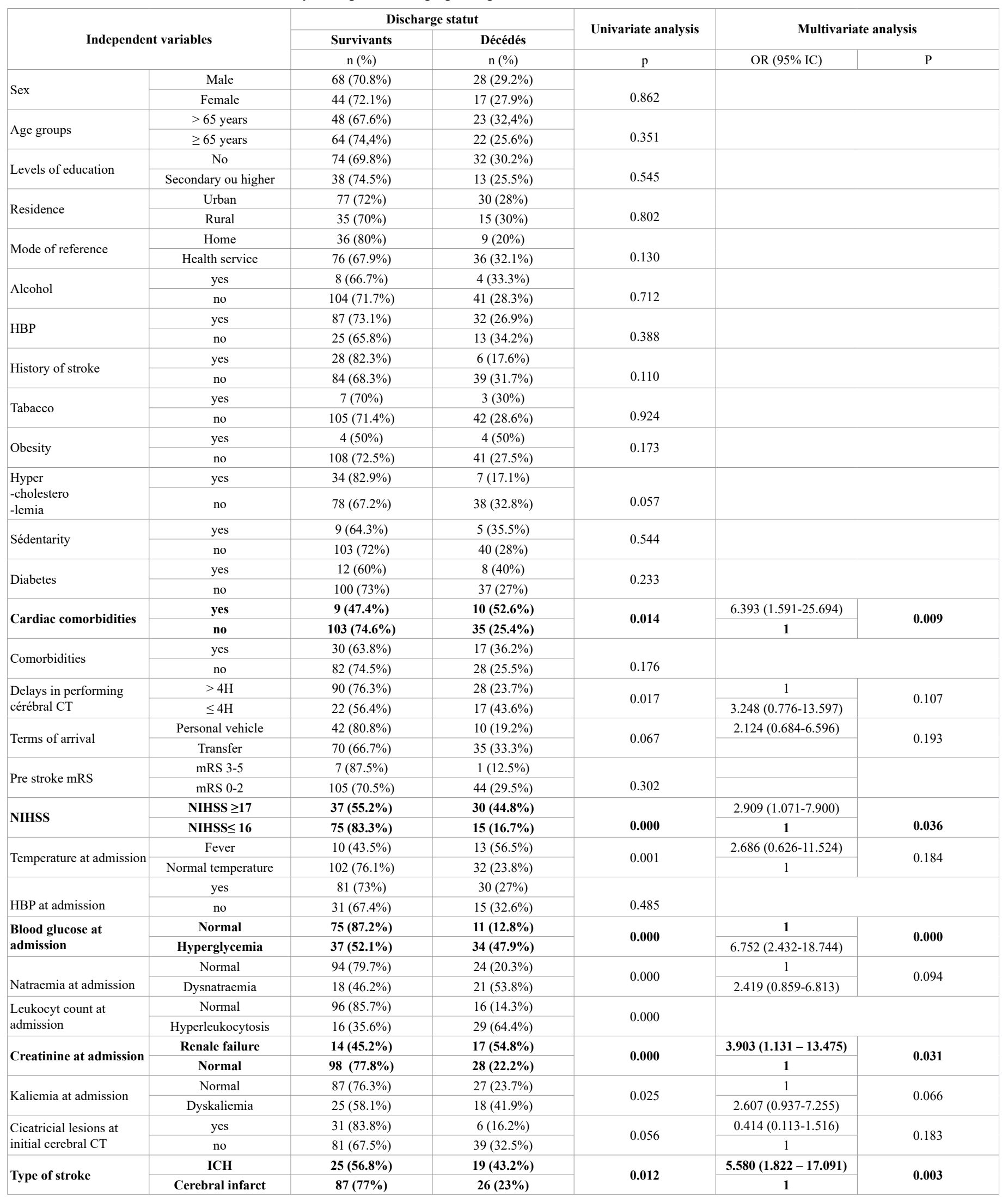




\begin{tabular}{|c|c|c|c|c|c|c|}
\hline \multirow{2}{*}{$\begin{array}{l}\text { Leucoaraiosis at brain } \\
\text { scan }\end{array}$} & yes & $52(78.8 \%)$ & $14(21.2 \%)$ & \multirow{2}{*}{0.080} & & \\
\hline & no & $60(65.9 \%)$ & $31(34.1 \%)$ & & & \\
\hline \multirow{2}{*}{ Swallowing disorders } & yes & $46(53.5 \%)$ & $40(46.5 \%)$ & \multirow{2}{*}{0.000} & & \\
\hline & no & $66(93 \%)$ & $5(7 \%)$ & & & \\
\hline \multirow{2}{*}{ Admission times } & $>24 \mathrm{~h}$ & $23(88.5 \%)$ & $3(11.5 \%)$ & \multirow{2}{*}{0.035} & & \\
\hline & $\leq 24 \mathrm{~h}$ & $89(67.9 \%)$ & $42(32.1 \%)$ & & & \\
\hline \multirow{2}{*}{ GCS at admission } & $<=8$ (coma) & $9(47.4 \%)$ & $10(52.6 \%)$ & \multirow{2}{*}{0.014} & $3.160(0.959-10.904)$ & \multirow{2}{*}{0.054} \\
\hline & $>9$ & $103(74.6 \%)$ & $35(25.4 \%)$ & & 1 & \\
\hline
\end{tabular}

We have found as others $[13,22,27,35]$ that ICHs were an independent predictive factor of early post-stroke intra-hospital mortality. It is universal that ICHs, compared to cerebral infarcts, have a higher mortality rate [18,27]. Indeed, they are more often accompanied by an early alteration of vigilance, or even coma, due to a more frequent and earlier intracranial hypertension.

We have shown, like others [25,36-38], that cardiac comorbidities were also an independent predictive factor of intra-hospital mortality, due to the often advanced age of these patients, the usual clinical extent and severity of cardiac embolism, of a high rate of early cardiac mortality $[36,39,40]$. Specialized cardiac assessment and management is required in patients with acute stroke associated with cardiac comorbidities [36].

Finally, we identified renal insufficiency at admission as an independent predictive factor of intra-hospital mortality per stroke, in agreement with some publications [41-44]. This could be explained by an increase in the susceptibility of these patients to infections and a greater frequency of fatal cardiac complications secondary to metabolic disorders [45].

Other independent predictors of intra-hospital stroke mortality, identified by some authors, such as sex and especially age [27], severe initial hypertension [22], history of stroke [10,22], were not found in our study.

However, our study suffered from a number of shortcomings: the fact that patients were hospitalized more than 72 hours after the onset of stroke significantly reduced the number of patients, the late admission of some patients (16.6\%) did not make it possible to obtain the early data of the beginning of the stroke and finally we did not have the neuroradiological data for the intra-hospital follow-up of our patients because of the non-accessible financial possibility of the cerebral scanner. However, these shortcomings have not hindered the relevance of our study.

\section{Conclusion}

Intra-hospital mortality of stroke remains high in Sub-Saharan Africa, ranging from $25 \%$ to $45 \%$, and the hospital mortality rate of $28.7 \%$ in our study falls within this range. The initial clinical severity of the stroke and initial hyperglycemia, indicative of the extent of neurological damage caused by stroke, cardiac comorbidities due to the usual severity of cardio-embolic infarction and resulting cardiac mortality, the haemorrhagic nature of stroke due to high coma propensity due to intracranial reaction hypertension and renal failure by associated cardiac and infectious complications are the independent predictive factors of early post-stroke deaths. Strategies to reduce early stroke mortality should include screening, monitoring and management of subgroups of patients most at risk of early death, specifically those with hyperglycemia and / or severe neurological deficit and / or coma at the outset, as well as early detection and specialized cardiac and / or nephrological management of patients with cardiac or renal comorbidities. These strategies should also include the prevention and early treatment of intracranial hypertension, ideally in SUs that have proven to be highly effective in reducing mortality and stroke disability around the world.

\section{References}

1. Feigin VL, Lawes CM, Bennett DA, Barker Collo SL, Parag V (2009) Worldwide stroke incidence and early case fatality reported in 56 population based studies: a systematic review. Lancet Neurol 8: 355-369. [Crossref]

2. Feigin VL, Forouzanfar MH, Krishnamurthi R, Mensah GA, Connor M, et al. (2014) Global and regional burden of stroke during 1990-2010: findings from the Global Burden of Disease Study 2010. Lancet 383: 245-254. [Crossref]

3. Heuschmann PU, Kolominsky Rabas PL, Misselwitz B, Hermanek P, Leffmann C, et al (2004) Predictors of in hospital mortality and attributable risks of death after ischemic stroke: the German Stroke Registers Study Group. Arch Intern Med 164: 1761-1768. [Crossref]

4. Saposnik G, Hill MD, O’Donnell M, Fang J, Hachinski V, et al. (2008) Variables associated with 7-day, 30-day, and 1-year fatality after ischemic stroke. Stroke 39: 2318-2324. [Crossref]

5. Smith EE, Shobha N, Dai D, Olson DM, Reeves MJ, et al. (2010) Risk score for in hospital ischemic stroke mortality derived and validated within the Get With The Guidelines-Stroke Program. Circulation 122: 1496-1504. [Crossref]

6. OCDE (2015) Cardiovascular Disease and Diabetes: Policies for Better Health and Quality of Care, OECD Health Policy Studies, Éditions OCDE, Paris, France.

7. Flower O, Smith M (2011) The acute management of intracerebral hemorrhage. Curr Opin Crit Care 17: 106-114. [Crossref]

8. Zahuranec DB, Lisabeth LD, Sánchez BN, Smith MA, Brown DL, et al. (2014) Intracerebral hemorrhage mortality is not changing despite declining incidence. Neurology 82: 2180-2186. [Crossref]

9. Connor MD, Thorogood M, Modi G, Warlow CP (2007) The burden of stroke in SubSaharan Africa. Am J Prev Med 33: 172-173. [Crossref]

10. Touré K, Diagne SN, Seck LB, Sow A, Ndiaye M, et al. (2010) Predictive factors of stroke mortality at the Fann neurological clinic in Dakar. Afr J Neurol Sci 29: 29-36.

11. Ossou-Nguiet P M, Gombet T, Ossil-Ampion M, Ellenga-Mbolla B F, Otiobanda G F, et al. (2013) Stroke mortality factors at the Brazzaville University Hospital. Rev Afr Anesth Méd Urg Tome 18. [Crossref]

12. Kuate-Tegueu (2016) Determinants of Mortality by Cerebral Vascular Accident in a Douala Hospital in Cameroon. Health Sci Dis 17: 1-6.

13. Mapoure NY, Tchaleu Nguenkam CB, Mbatchou Ngahane HB, Dzudie A, Coulibaly A, et al. (2014) Predictors of In-Hospital Mortality for Stroke in Douala, Cameroon Hindawi Publishing Corporation/Stroke Research and Treatment: 6.

14. Nakibuuka J, Sajatovic M, Nankabirwa J, Ssendikadiwa C, Furlan AJ, et al. (2015) Early mortality and functional outcome after acute stroke in Uganda: prospective study with 30 day follow-up. Springerplus 4: 450. [Crossref]

15. Ekeh B, Ogunniyi A, Isamade E, Ekrikpo U (2015) Stroke mortality and its predictors in a Nigerian teaching hospital. Afr Health Sci15: 74-81. [Crossref]

16. Ogun SA, Ojini FI, Ogungbo B, Kolapo KO, Danesi MA (2005) Stroke in south west Nigeria: a 10-year review. Stroke 36: 1120-1122. [Crossref]

17. Mudzi W, Stewart A, Musenge E (2012) Case fatality of patients with stroke over a 12-month period post stroke. S Afr Med J 102: 765-767. [Crossref]

18. Sagui E (2007) Stroke in sub-Saharan Africa. Med Trop (Mars) 67: 596-600. [Crossref]

19. Ovbiagele B, Buck BH, Liebeskind DS, Starkman S, Bang OY, et al. (2008) Prior antiplatelet use and infarct volume in ischemic stroke. J Neurol Sci 264: 140-144. [Crossref] 
20. Selim M, Savitz S, Linfante I, Caplan L, Schlaug G (2005) Effect of pre-stroke use of ACE inhibitors on ischemic stroke severity. BMC Neurol 5: 10. [Crossref]

21. Mills A (2014) Health care systems in low- and middle-income countries. $N$ Engl J Med 370: 552-557. [Crossref]

22. Femi OL, Mansur N (2013) Factors associated with death and predictors of one-month mortality from stroke in Kano, Northwestern Nigeria. J Neurosci Rural Pract 4: S5661. [Crossref]

23. Navarrete-Navarro P, Rivera-Fernández R, López-Mutuberría MT, Galindo I, Murillo $\mathrm{F}$, et al. (2003) Outcome prediction in terms of functional disability and mortality among ICU-admitted severe stroke patients: a prospective epidemiological study in the south of the European Union (Evascan Project, Andalusia, Spain). Intensive Care Med 29:1237-1244. [Crossref]

24. Rordorf G, Koroshetz W, Efird JT, Cramer SC (2000) Predictors of mortality in stroke patients admitted to an intensive care unit. Crit Care Med 28: 1301-1305. [Crossref]

25. Saposnik G, Kapral MK, Liu Y, Hall R, O’Donnell M, et al. (2011) Investigators of the Registry of the Canadian Stroke Network and the Stroke Outcomes Research Canada (SORCan) Working Group. I Score A Risk Score to Predict Death Early After Hospitalization for an Acute Ischemic Stroke. Circulation 123: 739-749. [Crossref]

26. Garbusinski JM, van der Sande MAB, Bartholome EJ, le Dramaix M, et al. (2005) Stroke Presentation and Outcome in Developing Countries. A Prospective Study in The Gambia. Stroke 36: 1388-1393. [Crossref]

27. de Peretti C (2015) The risk of death one year after a stroke. Studies and Results, Directorate of Research, Studies, Evaluation and Statistics (DREES).

28. Owolabi MO, Bower JH, Ogunniyi A (2007) Mapping Africa's way into prominence in the field of neurology. Arch Neurol 64: 1696-1700. [Crossref]

29. Gilmore RM, Stead LG (2006) The role of hyperglycemia in acute ischemic stroke. Neurocrit Care 5: 153-158. [Crossref]

30. Gray CS, Hildreth AJ, Alberti GKMM, O'Connell JE (2004) Poststroke hyperglycemia natural history and immediate management. Stroke 35: 122-126. [Crossref]

31. Parsons MW, Barber PA, Desmond PM, Baird TA, Darby DG, et al. (2002) Acute hyperglycemia adversely affects stroke outcome: a magnetic resonance imaging and spectroscopy study. Ann Neurol 52: 20-28. [Crossref]

32. Stead LG, Gilmore RM, Bellolio MF, Shaily M, Bhagra, et al. (2009) Hyperglycemia as An Independent Predictor of Worse Outcome in Non-diabetic Patients Presenting with Acute Ischemic Stroke. Neurocrit Care 10: 181-186. [Crossref]

33. Koivunen R-J, Satop J, Meretoja A (2015) Incidence, risk factors, etiology, severity, and short-term outcome of nontraumatic intracerebral hemorrhage in young adults. Eur J Neurol 22: 123-132. [Crossref]
34. Passero S, Ciacci G, Ulivelli M (2003) The influence of diabetes and hyperglycemia on clinical course after intracerebral hemorrhage. Neurology 61: 1351-1356. [Crossref]

35. Jover-Saenz A, Porcel-Perez JM, Vives-Soto M, Rubio-Caballero M (1999) Epidemiology of acute cerebrovascular disease in Lleida from 1996-1997. Predictive factors of mortality at short and medium term. Rev Neurol 28: 941-948. [Crossref]

36. Kimura K, Minematsu K, Yamaguchi Y, Japan Multicenter Stroke Investigators'Collaboration (J-MUSIC) (2005) Atrial fibrillation as a predictive factor for severe stroke and early death in 15831 patients with acute ischaemic stroke. $J$ Neurol Neurosurg Psychiatry 76: 679-683. [Crossref]

37. Sandercock P, Bamford J, Dennis M (1992) Atrial fibrillation and stroke: prevalence in different types of stroke and influence on early and long term prognosis (Oxfordshire community stroke project). BMJ 305: 1460-1465. [Crossref]

38. Wong KS (1999) Asian Acute Stroke Advisory Panel. Risk Factors for Early Death in Acute Ischemic Stroke and Intracerebral Hemorrhage. A Prospective Hospital-Based Study in Asia. Stroke 30: 2326-2330. [Crossref]

39. Kaarisalo MM, Immonen-Raiha P, Marttila RJ (1997) Atrial fibrillation and stroke. Mortality and causes of death after the first acute ischemic stroke. Stroke 28: 311-315. [Crossref]

40. Gustafsson C, Britton M (1991) Pathogenetic mechanism of stroke in non-valvular atrial fibrillation: follow-up of stroke patients with and without atrial fibrillation. $J$ Intern Med 230: 11-16. [Crossref]

41. Dong K, Huang X, Zhang Q, Yu Z, Ding J, et al. (2017) A lower baseline glomerularfiltration rate predicts high mortality and newly cerebrovascular accidents in acute ischemic stroke patients. Medicine 96: 5(e5868). [Crossref]

42. El Husseini N, Fonarow GC, Smith EE, Ju C, Schwamm LH, et al. (2016) Renal Dysfunction Is Associated With Poststroke Discharge Disposition and In-Hospital Mortality. Stroke 48: 327-334. [Crossref]

43. Elyar Sadeghi-Hokmabadi E, Bas DF, Farhoudi M, Taheraghdam A, Oskouei DS, et al (2017) Renal Dysfunction Is an Independent Risk Factor for Poor Outcome in Acute Ischemic Stroke Patients Treated with Intravenous Thrombolysis: A New Cutoff Value. Stroke Res Treat : 6. [Crossref]

44. Hojs Fabjan T, Hojs R, Tetickovic E, Pecovnik Balon B (2007) Ischaemic stroke-impact of renal dysfunction on in-hospital mortality. Eur J Neurol 14: 1351-1356. [Crossref]

45. Hao Z, Yang C, Liu M, Wu B (2014) Renal dysfunctionand thrombolytic therapy in patients with acute ischemic stroke: a systematic review and meta-analysis. Medicine 93: e286. [Crossref]

Copyright: (C2017 Labodi LD. This is an open-access article distributed under the terms of the Creative Commons Attribution License, which permits unrestricted use, distribution, and reproduction in any medium, provided the original author and source are credited. 